\title{
Simulation of single-phase ground short circuit protection device
}

\author{
Aliya Zhumadirova ${ }^{1, *}$, Vadim Markovskiy ${ }^{1}$, and Aleksandr Kislov ${ }^{1}$ \\ ${ }^{1}$ NJSC Toraighyrov University, 140008 Pavlodar, Kazakhstan
}

\begin{abstract}
The most common type of short circuit in a three-phase system is a single-phase-to-ground short circuit, accounting for 70 to $90 \%$ of electrical faults. In this paper simulation of the protective device from single-phase-to-ground short circuit with automatic change of current setting in electrical networks of 6-10 $\mathrm{kV}$ voltage on the basis of Coloured Petri Net is considered. The complexity of elaborated technical systems makes the problem of their modelling actual at the stage of development with the purpose of obtaining estimations of prospective and achievable characteristics. Now the modelling theory of the dynamic discrete systems, based on the formalism of Coloured Petri Nets has a wide application. Petri Net, describing the device operation, was designed and analysed using CPN Tools Programm. The resulting Petri net is correct, as it is live, reversible and safe. Thereby designed device can provide the selective protection from singlephase-to-ground short circuit.
\end{abstract}

\section{Introduction}

The most widespread kind of damages in electrical networks of $6-10 \mathrm{kV}$ voltage is single-phase-to-ground short circuit (SPGSC) comprising 70-90\% of the general number of electrical damages. In order to increase efficiency of power supply system the device for centralized protection from single-phase-to-ground short circuit is developed. To define structural and behavioral properties of the developed device is necessary to build a model.

SPGSC quite often leads to major accidents accompanied by significant losses, and to the following negative consequences [1-3]:

- occurrence in the electrical network of 2,4-3,5 multiple surge voltages in comparison with phase voltage, that can lead to insulation damage of undamaged phases and to the transition of SPGSC in a two-place or double short circuit to ground, which is close to two-phase short circuits;

- occurrence of ferroresonance phenomenon, resulting in frequent voltage transformers falling out, sometimes in the damage of underloaded power transformers working in a mode near to idling;

- the danger of electrical shock of people under a broken wire and its falling on the ground;

- insulation damage of the motor stator windings, which leads to the occurrence of dangerous coil or phase-to-phase short circuits;

- increased risk of injury to people by step voltage or touch voltage;

- fire probability, for example, in cells of the complete switchgear centre because of high-temperature arc in the location of SPGSC.
Frequently, the search of the damaged line under SPGSC is conducted by serial switchings-off of lines and can take some hours. In spite of the fact that many works are devoted to studying and development of protection from SPGSC, still there are no calculation methods of the current setting and sensitivity testing of directional current protections from SPGSC. As a result of an incorrect setting the efficiency of sensitive protection from SPGSC substantially decreases. As a rule, even knowing the relay setting, it is impossible to calculate precisely the operating current especially in the presence of several feeders in the load node. In practice, it is necessary to specify the operating current by actual test.

At present, difficulties arise with the choice of settings and checking the sensitivity of the zero sequence current protection in the overhead lines of the electrical network. As sources of zero sequence current it is often necessary to use a three-transformer filter, the imbalance at its output can be rather high, which causes the necessity of choosing a high operating current. Under SPGSC on overhead lines with falling wire to ground in a fault location there can be a great transient resistance, of the order of 5-7 $\mathrm{kOhm}$ and more, which leads to essential reduction of zero sequence currents and voltage, and reduces the protection sensibility of the damaged line.

As a rule, the character of the processes arising in an electrical network under SPGSC to a great extent depends on the mode of neutral ground connection. Now, the majority of experts suggests rejecting systems with isolated neutral and gives preference to systems with resonant, high-resistance or combined (resonanthigh resistance) neutral. However, under the conditions

\footnotetext{
* Corresponding author: zhumadirova.a@teachers.tou.edu.kz
} 


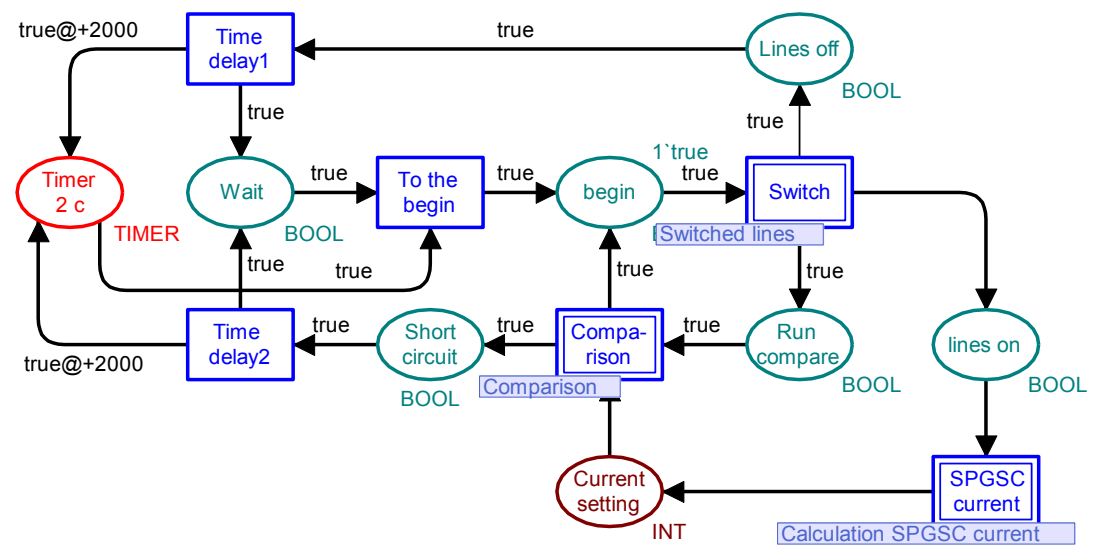

Fig. 1. The main Petri Net of the protection device from single-phase-to-ground short circuit with automatic change of current setting.

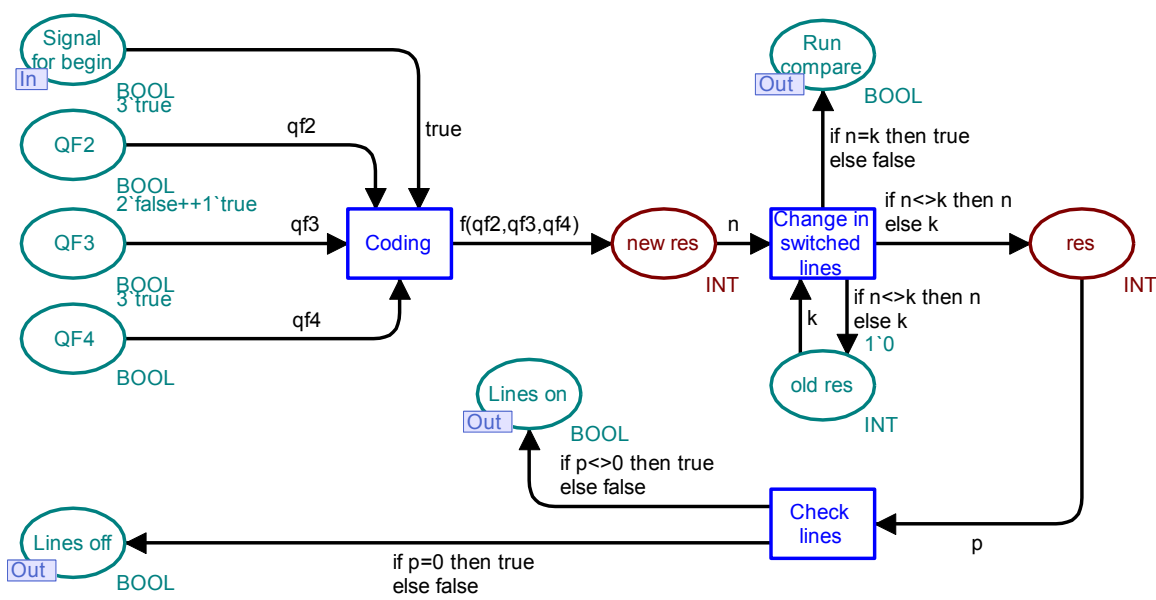

Fig. 2. Switched lines subnet.

of preservation of neutral mode, the issue of developing a selective protection from SPGSC remains urgent.

\section{Materials and methods}

The complexity of elaborated technical systems makes the problem of their modelling actual at the stage of development with the purpose of obtaining estimations of prospective and achievable characteristics. Now, the modelling theory of the dynamic discrete systems, based on the formalism of Color Petri Nets (CPN), has a wide application [4-16]. The search of description methods of parallel systems has led to treating CPN as the most acceptable tool for a visual presentation of the multitude of internal states and conditions of their change.

$\mathrm{CPN}$ is a graph-oriented language for designing, description, imitation and control of distributed and parallel systems. The graphical primitives show the course of the process, and constructions of a special language simulate the necessary data processing. Unlike the "classical" Petri Nets, in CPN an important role is played by data typification based on the conception of colour multitude, which is similar to the type in declarative programming languages. Accordingly, for manipulation with colour, they use variables, functions and other elements, known from the programming languages. The theory of CPN is close to the structural methods of system simulation; however, unlike many of them, it is based on a well-developed mathematical instrument and, consequently, allows carrying out analytical and imitating researches [17-19].

The device's operation principle consists in [20]:

- automatic connection additional capacitive susceptance to one of phases of electrical network;

- reading out a line voltage, phase-to-ground voltage and zero-sequence voltage from the voltage transformer, their conversion to digital codes, on the basis of which the value of SPGSC current is calculated, keeping in data memory;

- disconnection additional capacitive susceptance from electrical network;

- reading out from current transformers of each line value of proceeding currents, converting to a digital code;

- the values of line currents are comparing with the calculated SPGSC current;

- the line where the current is commensurable to SPGSC current is disconnecting;

- changing of current setting after quantity change of working lines in the load node. 


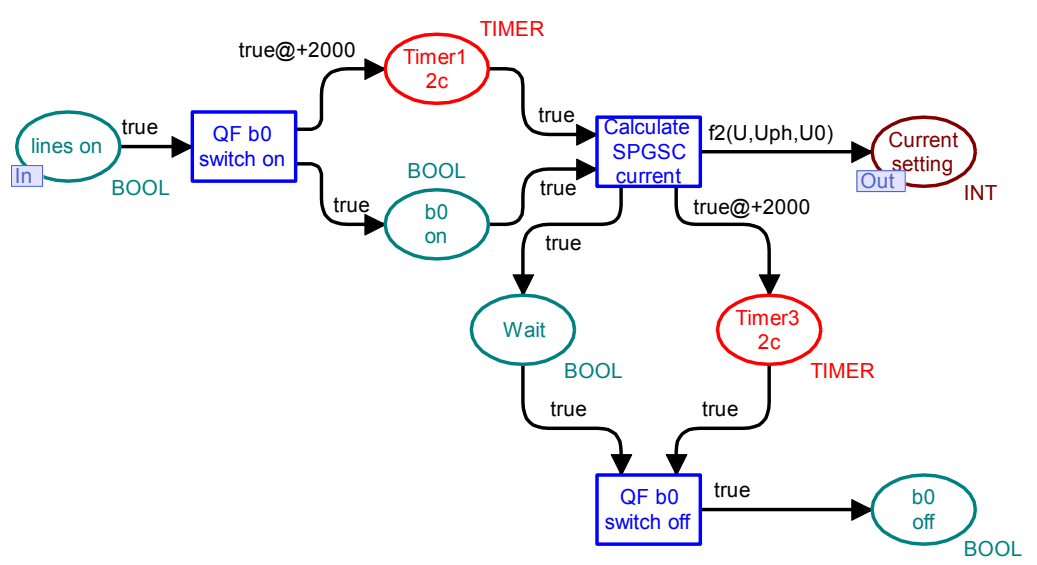

Fig. 3. Calculation SPGSC current subnet.

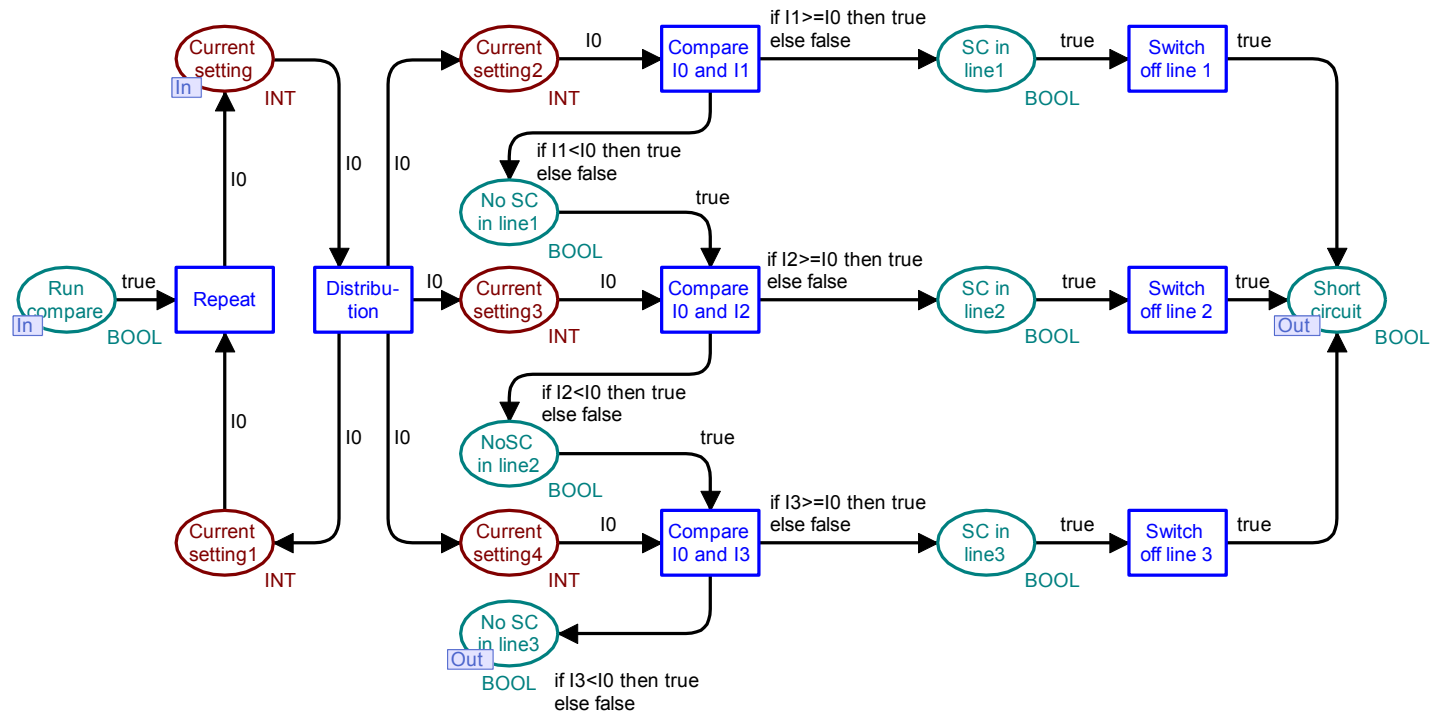

Fig. 4. Comparison subnet.

\section{Results and discussions}

The main CPN of the device is given in Fig. 1 [21]. It shows that we have three subnets - Switched lines, Calculation of SPGSC current and Comparison. Place Begin has one token in the initial state. The token value belongs to the type BOOL and it represents input buffer of the Switched lines subnet, which is given in Fig. 2.

Places $Q F 2, Q F 3, Q F 4$ represent switch position relays and have three tokens in initial state with BOOL type: "true" means line is switch on and "false" - line is switch off. Coding transition converts Boolean signals in integral number, which is placed in New res. Change in switched lines transition compares two results, defines if some of lines was connected or disconnected from the electrical network and stores new result. If there are no switched-on lines the Check lines transition sends signal to output buffer Lines off, otherwise - to output buffer Lines on. Lines on also represents input buffer of the Calculation SPGSC current subnet, which is given in Fig. 3.
QF b0 switch on transition sends a connection signal of additional capacitive susceptance $b_{0}$ to the electrical network. Timerl serves as time delay. Signals of phaseto-ground voltage $U_{P H}$, line voltage $U_{L I N}$ and zerosequence voltage $U_{0}$ modules are read out from secondary of the voltage transformer. Calculate SPGSC current transition estimates the value of SPGSC current (current setting) by expression [22]

$$
I_{0}=\frac{U_{L I N} U_{P H}}{U_{0}} b_{0} .
$$

Calculated value of SPGSC current places in Current setting output buffer. $Q F$ bo switch off transition disconnects additional capacitive susceptance $b_{0}$ from the electrical network. On Fig. 4 Comparison subnet is given. Signals of the proceeding currents in the off-going lines are read out from the secondary winding of the current transformers. Compare IO and I1, Compare IO and $I 2$ and Compare $I 0$ and I3 transitions compare the values of the currents in the off-going lines with the calculated SPGSC current. If insulation of any line in relation to the ground is damaged, Switch off line 1, 
Switch off line 2 or Switch off line 3 transitions disconnect the damaged line from the three-phase electrical network and blocks the protection of other lines to avoid misoperation. In this case Short circuit place is input buffer for main net, shown on fig. 1. Time delay 2 transition is needed for delay while damaged line is disconnecting. Then pass to the beginning. In case, in all the connections current modules are not commensurable with the setting, Compare IO and I3 transition sends signal to output buffer No SC in line3, which is input buffer for Switched lines subnet (fig. 2). If any line hasn't been connected or disconnected from electrical network Change in switched lines transition sends signal to Run compare output buffer to repeat compare (fig. 4).

Based on the result of this analysis, we can say that designed Petri net is correct, as it is live, reversible (dead-end free) and safe (one token in any place).

\section{Conclusion}

Modeling of the device for centralized protection from single-phase-to-ground short circuit by means of CPN allows determining the structure and behavior of the device. To conduct such research, it is necessary to perform the analysis of the obtained Petri Nets characteristics, and from the properties of the modeling Petri Net pass on to the properties of the real system. Application of the given method allows to realize the centralized protection of lines from single phase ground short circuit with automatic change of current setting, provides the protection selectivity from SPGSC and increases the reliability and electrical safety level in the operation of electrical installations.

\section{References}

1. A.A. Krasnykh, I.L. Krivoshein, A.L. Kozlov, Research of single-phase faults in 6-35 KV branched overhead distribution network, 2017 International Conference on Industrial Engineering, Applications and Manufacturing (ICIEAM), $\quad 1-4 \quad$ (2017) DOI: 10.1109/ICIEAM.2017.8076252

2. D.N. Pelenev, B.N. Abramovich, K.V. Babyr, Increase Effectiveness Functioning of Protection against Single-Phase Ground Fault Electrical Networks Medium Voltage, 2020 IEEE Conference of Russian Young Researchers in Electrical and Electronic Engineering (EIConRus), 1291-1296 (2020) DOI: 10.1109/EIConRus49466.2020.9039469

3. Dongping Xiao, Tao He, Ruiyang Xiao, Xuefei $\mathrm{Du}$, Segment location for single-phase-to-ground fault in neutral non-effectively grounded system based on distributed electric-field measurement, Electric Power Systems Research, 184, 106321 (2020) DOI: 10.1016/j.epsr.2020.106321

4. Jing Sun, Shi-Yin Qin, Yong-Hua Song, Fault diagnosis of electric power systems based on fuzzy Petri nets, IEEE Transactions on Power
Systems, 19 (4), 2053-2059 (2004) DOI: 10.1109/TPWRS.2004.836256

5. B. Xu, X. Yin, X. Yin, Y. Wang, S. Pang, Fault Diagnosis of Power Systems Based on Temporal Constrained Fuzzy Petri Nets, IEEE Access, 7, 101895-101904 (2019) DOI: 10.1109/ACCESS.2019.2930545

6. V. Calderaro, C.N. Hadjicostis, A. Piccolo, P. Siano, Failure Identification in Smart Grids Based on Petri Net Modeling, IEEE Transactions on Industrial Electronics, 58 (10), 4613-4623 (2011) DOI: $10.1109 /$ TIE.2011.2109335

7. A.I. Pózna, A. Fodor, M. Gerzson, K.M. Hangos, Colored Petri net model of electrical networks for diagnostic purposes, IFAC-PapersOnLine, 51 (2), 260-265 (2018) DOI: 10.1016/j.ifacol.2018.03.045

8. Yan Zhang, Yong Zhang, Fushuan Wen, Chi Yung Chung, Chung-Li Tseng, Xiaoyi Zhang, Fei Zeng, Yubo Yuan, A fuzzy Petri net based approach for fault diagnosis in power systems considering temporal constraints, International Journal of Electrical Power \& Energy Systems, 78, 215-224 (2016) DOI: 10.1016/j.ijepes.2015.11.095

9. Abouelabbas Ghanaim, Georg Frey, Markov Modeling of Delays in Networked Automation and Control Systems Using Colored Petri Net Models Simulation, IFAC Proceedings Volumes, $44 \quad$ (1), 2731-2736 (2011) DOI: 10.3182/20110828-6-IT-1002.02322

10. Brigitta Márczi, Adrien Leitold, Miklós Gerzson, Diagnosis of Technological Systems based on their Coloured Petri Net Model, IFAC Proceedings Volumes, 45 (2), 1201-1206 (2012) DOI: $10.3182 / 20120215-3-A T-3016.00213$

11. Chuanlai Yuan, Yongyi Liao, Lingshuang Kong, Huiqin Xiao, Fault diagnosis method of distribution network based on time sequence hierarchical fuzzy petri nets, Electric Power Systems Research, 191, 106870 (2021) DOI: 10.1016/j.epsr.2020.106870

12. Hui Ren, Zengqiang Mi, Power system fault diagnosis modeling techniques based on encoded Petri nets, 2006 IEEE Power Engineering Society General Meeting, 6 (2006) DOI: 10.1109/PES.2006.1709100

13. R.O. González, G.G. González, J. Escobar, R.Y. Barazarte, Applications of Petri Nets in electric power systems, 2014 IEEE Central America and Panama Convention, 1-6 (2014) DOI: 10.1109/CONCAPAN.2014.7000469

14. Alessandro Giua, Manuel Silva, Petri nets and Automatic Control: A historical perspective, Annual Reviews in Control, 45, 223-239 (2018) DOI: $10.1016 /$ j.arcontrol.2018.04.006

15. C.A. Lopez de Alba, V.H. Ortiz Muro, A. Santoyo-Sanchez, Modelling Hybrid Petri Nets to analyze contingencies in Power Systems, 2015 North American Power Symposium (NAPS), 1-6 (2015) DOI: 10.1109/NAPS.2015.7335188 
16. G.P. Singh, Applications of Petri nets in electrical, electronics and optimizations, 2016 International Conference on Electrical, Electronics, and Optimization Techniques (ICEEOT), 2180-2184 (2016) DOI: 10.1109/ICEEOT.2016.7755078

17. J.L. Peterson, Petri net theory and the modeling of systems (Prentice-Hall, 290, 1981)

18. K. Jensen, Coloured Petri Nets - Basic Concepts, Analysis Methods and Practical Use (SpringerVerlag Berlin Heidelberg, 265, 1997) DOI: 10.1007/978-3-642-60794-3

19. K. Jensen, L.M. Kristensen, Coloured Petri Nets - Modelling and Validation of Concurrent Systems (Springer-Verlag Berlin Heidelberg, 384, 2009) DOI: $10.1007 / \mathrm{b} 95112$

20. B.B. Utegulov, A.B. Utegulov, A. Zhumadirova, Analysis of Petri Net of isolation state and singlephase ground short circuit control device in 6-10 $\mathrm{kV}$ electric networks, 4th International Scientific Colloquium Modelling for Material Processing (2006)

21. CPN Tools [Electronic resource] Available at: http://cpntools.org/

22. V.I. Shchutskii, B.B. Utegulov, Opredelenie toka zamykaniya na zemlyu $\mathrm{V}$ kar'ernykh setyakh napryazheniem vyshe $1000 \mathrm{~V}$, Dobycha uglya otkrytym sposobom, 6, 19-20 (1981) 